\title{
AUTOMATED MANUFACTURE OF GRID STIFFENED PANELS
}

\author{
J. MARCELO MÜLLER,* PETER NIJHUIS* \\ * Netherlands Aerospace Centre (NLR) \\ Voorsterweg 31, 8316 PR, Marknesse, The Netherlands \\ e-mail: peter.nijhuis@nlr.nl,web page: http://www.nlr.org
}

Key words: Composites Manufacturing, Orthogrid structure, Low cost tooling, Reusable vacuum bag, Conformal antenna, Automated Fibre Placement.

Abstract. Rib or grid stiffened structures have been investigated for decades, mainly for application in space structures.

Grid structures offer the possibility to develop more damage tolerant structures as the network of ribs can provide redundant load paths. In an ideal situation, an aircraft fuselage could have a load carrying grid with ribs that carry tensile, compressive and shear loads. The skin would only need to sustain the cabin pressure load.

Such a configuration could lead to significant weight reduction as a different design philosophy can be used. Typically, a damage tolerant design approach allowing a maximum of $3000 \mu$ strain is used for composite aircraft structures. Using a grid structure, a different approach could be developed for a design which could allow strains up to $6000 \mu$ strain.

Automated fibre placement has made composite grid structures an affordable option. However, they pose a major challenge in the development of tooling for cure due to their complex and sometimes irregular structure.

Within the European funded Horizon 2020 ACASIAS programme, square antenna elements are developed to be integrated in a composite aircraft fuselage panel. For this reason, an orthogrid stiffener pattern was chosen. As the skin of the panel must be transparent for the antenna signals, a glass fibre skin is required. A glass fibre skin to carry shear loads and carbon fibre ribs to carry compression and tension loads is therefore being developed to be able to meet structural and electromagnetic requirements.

This paper describes aspects of the ongoing development for the design, manufacture and testing of an orthogrid stiffened structure in an efficient way.

Simple alternating cutting of tapes at the crossing appear to be a suitable solution with a tensile stiffness reduction of $10 \%$ compared to the situation without cuts. These and other details and elements are tested to validate the final design.

The use of reusable silicone vacuum bags was investigated in order to replace complex metal tooling blocks. Besides saving on mould materials and machining, the use of a preshaped silicone vacuum bag is expected to reduce labour cost due to a decrease in handling of tooling blocks for positioning and cleaning.

The first trial indicates that good quality laminates can be obtained using reusable vacuum bags. More testing will be carried out to validate the concept for a large $3 \times 1.2 \mathrm{~m}$ panel. 


\section{INTRODUCTION}

The European Horizon 2020 ACASIAS programme aims to reduce energy consumption of future aircraft through research into integrated aero-structures with multifunctional capabilities.

One of the subjects within this project is improving aerodynamic performance by integrating antennas in the fuselage. This can not only reduce fuel consumption by reduction of aerodynamic drag, but can also make antennas less vulnerable to damage as well, as they do not protrude from the fuselage.

Antennas for communication with Ku-band geostationary satellites are used to provide internet access and entertainment on-board of aircraft. These used to be mechanically steered antennas. Currently, state-of-the-art is the use of hybrid antennas where the horizontal scanning is mechanical and the vertical scanning is electrical. ACASIAS aims to integrate a conformal phased array antenna into the fuselage, thus creating a conformal antenna. See Figure 1 for an illustration.

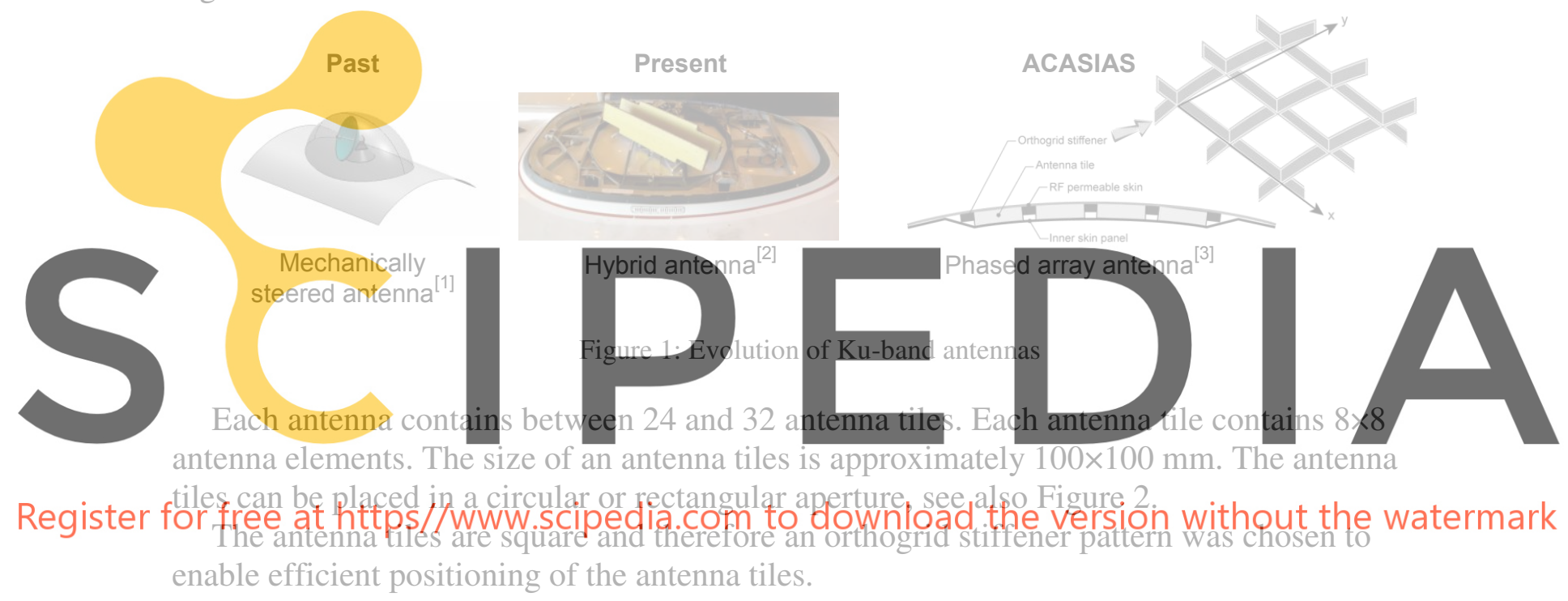

Rib stiffened structures, also referred to as grid or isogrid structures have been investigated for decades, mainly for application in space structures. Research was initially into metal grid structures and later into composite grid structures in both the USA and USSR ${ }^{[4]}$.

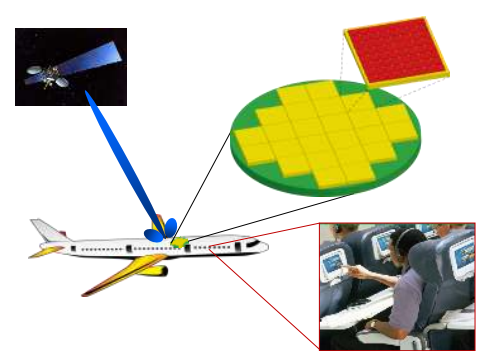

Figure 2: ACASIAS concept for airborne satellite communication

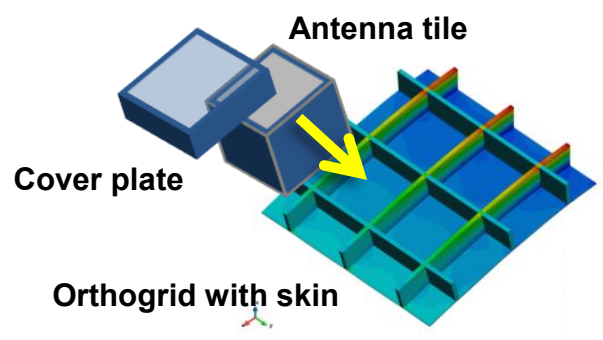

Figure 3: Basic manufacturing concept for fuselage panel 
Grid structures offer the possibility to develop more damage tolerant structures as the network of ribs can provide redundant load paths. In an ideal situation, an aircraft fuselage could have a load carrying grid with ribs that carry tensile, compressive and shear loads. The skin would only need to sustain the cabin pressure load.

Such a configuration could lead to significant weight reduction as a different design philosophy can be used. Typically, a damage tolerant design approach allowing a maximum of $3000 \mu$ strain is used for composite aircraft structures. Using a grid structure, a different approach could be developed for a design which could allow strains up to $6000 \mu$ strain.

The skin must be transparent for electromagnetic signals and carry shear loads. The orthogrid must carry compression and tension loads. A hybrid composite structure must therefore be employed to meet these structural and electromagnetic requirements. The requirements can met by using glass fibre reinforced composite for the skins and carbon fibre reinforced ribs. A fuselage panel section with this configuration is being designed, manufactured and tested. The panel section will be $3 \mathrm{~m}$ long and $1.2 \mathrm{~m}$ wide.

Automated fibre placement (AFP) has made composite grid structures an affordable option. However, they pose a major challenge in the development of tooling for cure due to their complex and sometimes irregular structure.

In the European Union sponsored project LOCOMACHS, it was demonstrated that high quality rib stiffeners can be manufactured in an automated way with relative ease. It is possible to simply place tapes on top of each other, thus forming a stack of thermoset tape that

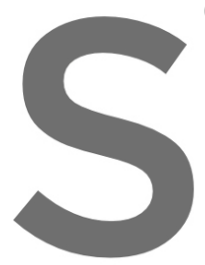
can be cured subsequen
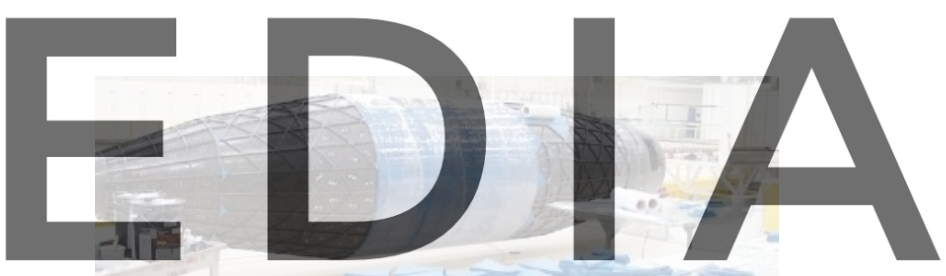

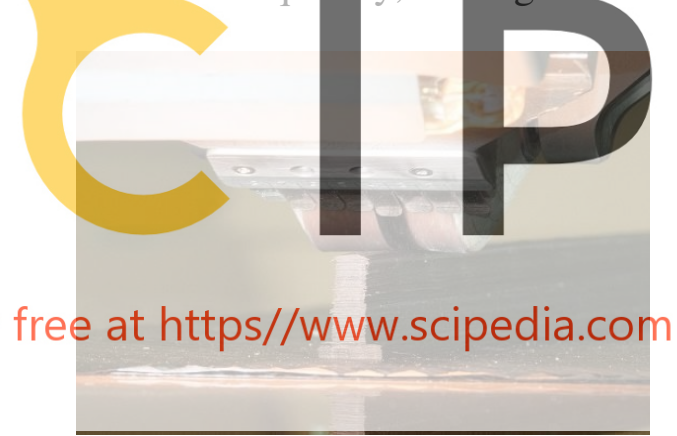

Figure 4: Manufacturing of ribs using automated fibre placement and thermoset material ${ }^{[5]}$
Figure 5: Advanced Grid-Stiffened Payload Fairing ${ }^{[6]}$

The deposition method using AFP does not require tooling. However, the cure of the component still requires an extensive tooling set, as tooling blocks must be inserted in all pockets to support the ribs during autoclave cure. This can lead to a large amount of complex tooling blocks. Figure 5 shows the tooling used for the cure of the Advanced Grid-Stiffened Composite Payload Fairing used on the Minotaur 1 launcher. It was developed by the Air Force Research Laboratory (AFRL), Boeing and Orbital Sciences Corp. This large amount of tooling blocks is undesirable, as it increases manufacturing risk and cost.

This paper will describe the ongoing development for the manufacture of an orthogrid stiffened fuselage panel using AFP in an efficient way. 


\section{ANALYSIS AND DESIGN}

A representative section of the fuselage of a regional aircraft was chosen for design, manufacture and testing.

Criteria for selection of the location of the antenna on the aircraft were:

- Aerodynamic and structural loads

- Field of view

- Available space

- Isolation from other antennas

- Location preferably in an area with low loads

For operation at high latitudes, two antennas may be needed, one at each side of the aircraft, to steer the beam to low elevations.

A compromise was reached between field of view vs expected stress and a forward panel with an angle of $30^{\circ}$ with regard to zenith was chosen. In Figure 6 the location is shown as a green patch.
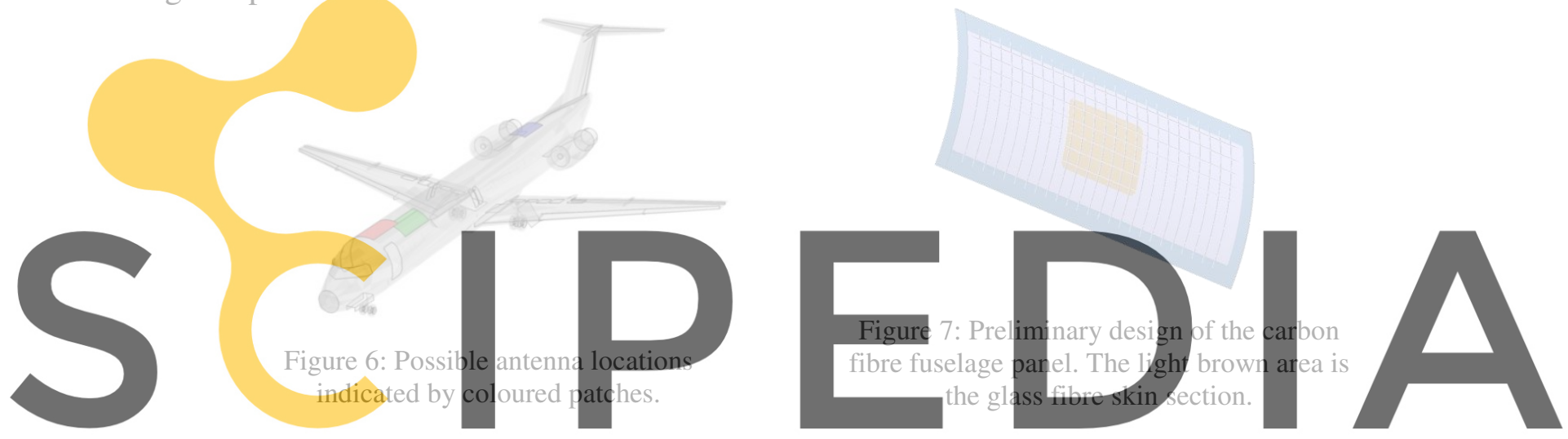

As the antenna signals must be able to pass through the skin, it is necessary to incorporate

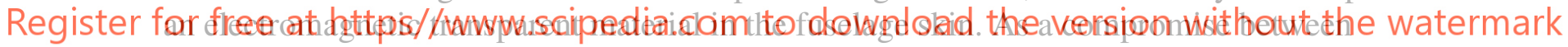
functionality and weight, it was chosen to include a section made out of glass fibre into the fuselage skin where the antenna tiles are to be placed.

One major issue with grid stiffened structures is the crossing of the grids. Simply laying crossing tapes on top of each other would lead to an excessive build-up at the crossing, leading to double the thickness of the ribs at the crossings. Spreading tows at the crossings could overcome this, but this will mean that at the crossings, the tape width will be double that of the regular width. The ideal situation would be that the height at the crossing is the same as the rest of the ribs. Preliminary analyses showed that alternate cutting of the tapes, leaving a gap for the transverse tape to pass, would result in a relative small decrease in stiffness at the crossings. This option was examined further.

Two cases were examined in comparison with the theoretical (ideal) case that both tapes would run continuously in both directions. One case is where the tapes are cut in an alternating fashion in each direction: the "real" case. The other is where one rib is kept intact at the crossing and the other rib is complete cut in two: the "worst" case. Figure 8 illustrates this. In Table 1, the results of the finite element analysis on representative material is given. As can be seen, for the alternate cuts, the loss in stiffness is limited. To maintain (buckling) stability, structural aerospace panel designs are normally stiffness driven. Considering the 
relative small drop in stiffness and enormous advantages in manufacturing, the alternating cut method was chosen to be implemented.

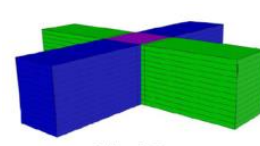

Ideal Case
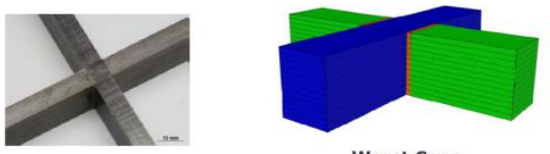

Worst Case
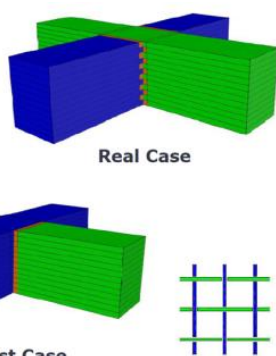

\begin{tabular}{rccc}
\hline & Tension & Bending & Pure shear \\
\cline { 2 - 4 } Ideal & 100 & 100 & 100 \\
Real & 98 & 96 & 97 \\
Worst & 90 & 91 & 94 \\
\hline
\end{tabular}

Table 1: Calculated stiffness reduction at crossing compared to the ideal case $(=100 \%)$. Source: CIMNE

Figure 8: Illustration of crossings and cuts.

The red areas represent cuts in the tape.

Source: CIMNE

The decrease in stiffness was compared with an actual test in tension. The average reduction in stiffness between UD coupon tests and actual crossing details with alternating cuts, i.e. the "real" case depicted in Figure 8. It appeared that the stiffness of the crossing was reduced to $90 \%$ of the UD coupon test value ("ideal" case in Figure 8). This value is lower than calculated. The calculated value assumes an ideal material, in reality, manufacturing deviations will occur which have to be accounted for. The decrease in stiffness was deemed acceptable and is used for the design of the larger panel.

A number of other d and peel strength betwee validate the finite elerne

Using representative loads, a design is being scale test of the $3 \times 1.2 \mathrm{~m}$ composite panel will
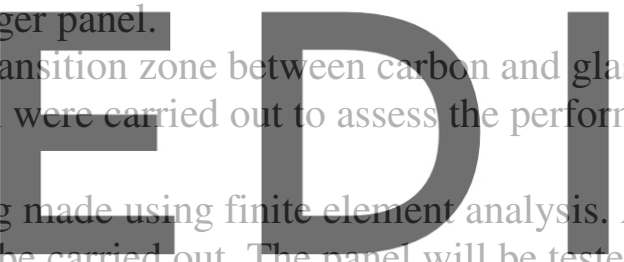
rig with which a combination of pressure, axial and circumferential loads can be applied.

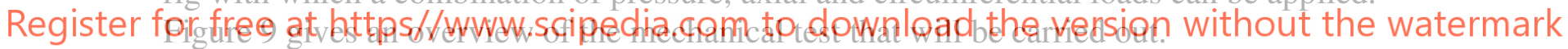
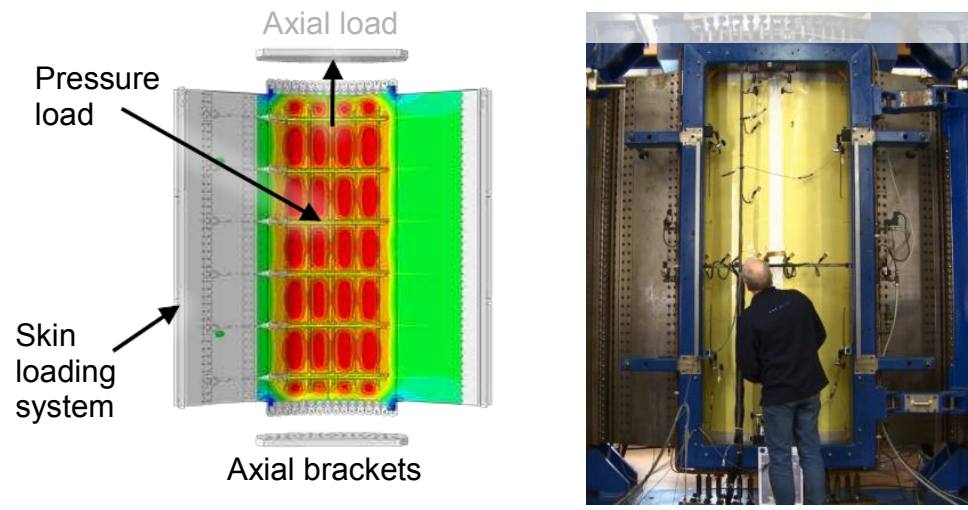

Figure 9: Overview of the panel test set-up. The actual test rig is shown on the right. 


\section{MANUFACTURING}

As discussed in the introduction, a large number of tooling blocks of different shapes and sizes are often necessary to cure an entire grid. The complexity is increased further when the panel is curved and the blocks must be placed on the inner side of the curvature. In this case the blocks must be collapsible and will consist of at least two parts. Although technically feasible, an alternative is desirable that requires less complex tooling and labour at the same or lower risk.

The option of employing reusable vacuum bags was explored to see if they can provide benefit over hard tooling. Reusable vacuum bags are used to replace single use bagging films during composite autoclave cure. They are usually made of silicone rubber and conform to the shape of the product that must be cured. Manufacturing of these vacuum bags is done by either brushing or spraying uncured silicone rubber on a plug in the shape of the final product followed by (often) room temperature cure. The bag obtained is resistant to temperatures of up to $260{ }^{\circ} \mathrm{C}$ and can be reused multiple times.

The height of ribs of the orthogrid under investigation is below $10 \mathrm{~mm}$. The grid itself is not a very complex shape in terms of geometry. A flexible vacuum bag could be easily placed over the product and removed afterwards. Compared to the material cost, the machining, cleaning, applying release agent and positioning of metal tooling blocks on the product, the silicone bag is an attractive option to save on material but also, perhaps even more important, on labour cost.

Trials are being carried out to investigate if a reusable silic ne bag shaped in the form of an orthogrid could be used for consolidation. The goal is not only to take advantage of the
flexibility of the bag to position and remgve it from the product, but to use t to simplify the
bag manufacture itself as well.
The intention is to manufacture a flat silicone bag that will be placed on the curved
product. The diameter of the fuselage under study is $3.3 \mathrm{~m}$. Consequently, the radius of the curvature is relatively large and the deformation of the flexible bag should be limited. In

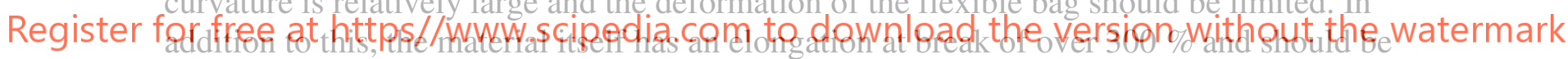
able to accommodate some deformation. In this way, it is possible to manufacture the vacuum bag in a low-cost way, without the need for a curved piug.

This concept is being tested on a small-scale first. A smaller panel was laid down using fibre placement for this purpose.

A simple plug was made using aluminium strips. Two-component silicone rubber was applied on the plug to form the bag (Figure 10). 


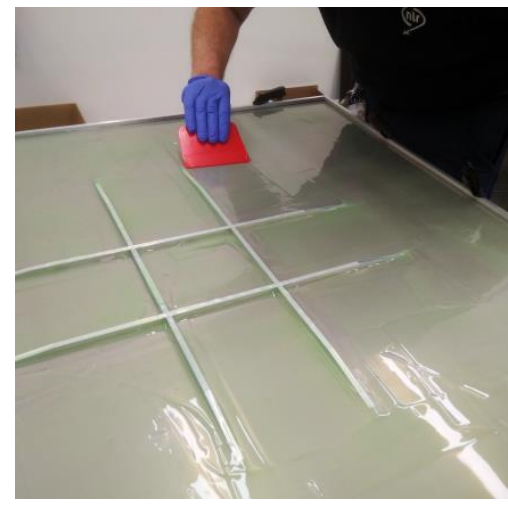

Figure 10: Silicone being applied to aluminium plug

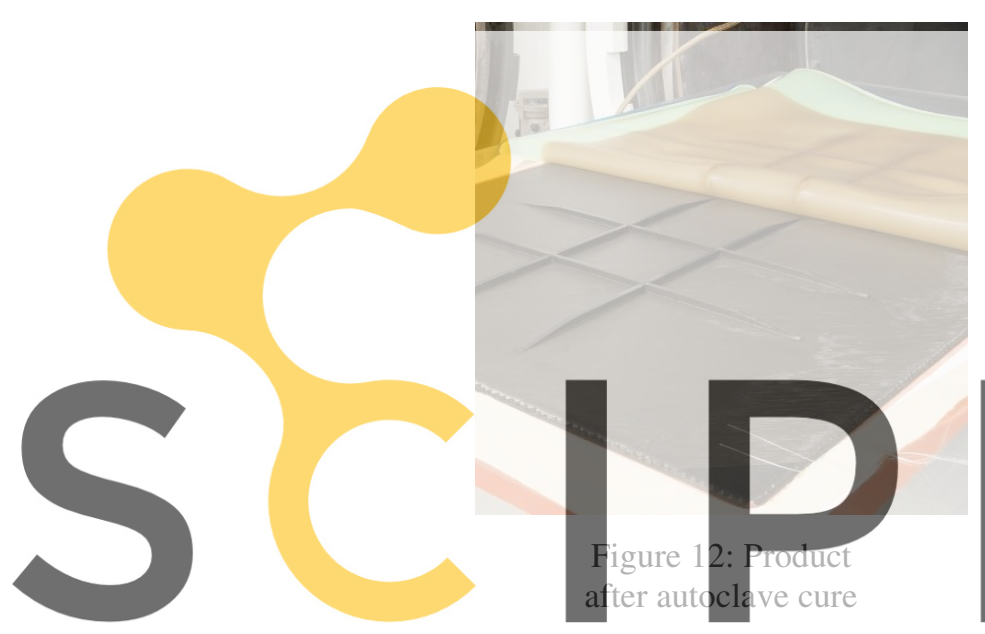

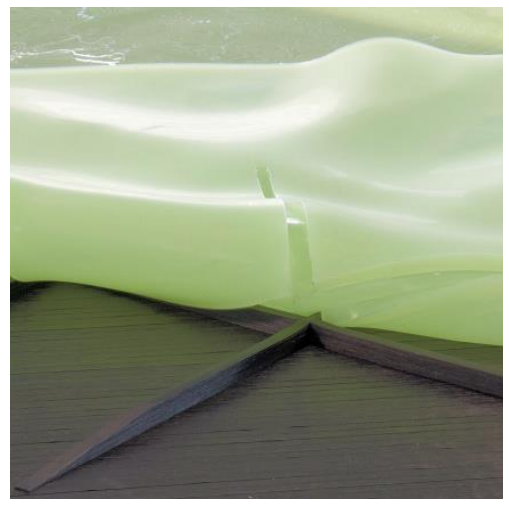

Figure 11: Placing vacuum bag over uncured product

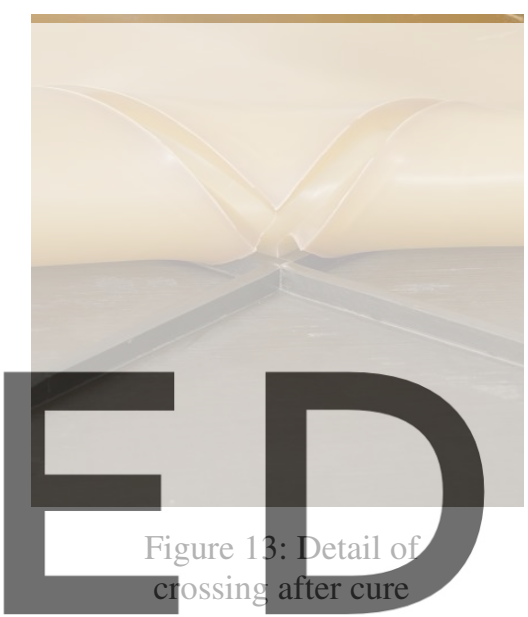

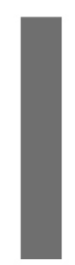

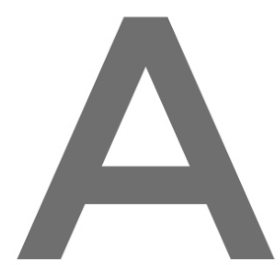

The fibre placed panel was bagged using the silicone bag (Figure 11) and subsequently Register forrqde(eiaturattpspdwrigur.s cippedia.com to download the version without the watermark

The resulting panel showed some surface roughness at the bagging surface. A C-scan confirmed that the skin laminate quality was good and the imperfections at the bagging side were only superficial. The cause of the surface roughness was not clear and is being investigated further. These first trials indicate that the use of reusable silicone vacuum bags could be an efficient alternative to more traditional moulding blocks.

\section{DISCUSSION AND CONCLUSIONS}

The goal of this study is to develop, design and a stiffened fuselage panel to incorporate an antenna array.

An orthogrid stiffening pattern was chosen to accommodate the square antenna tiles. The design, manufacture and test of a stiffened orthogrid panel are being investigated with an emphasis on a low-cost manufacturing solution.

Simple alternating cutting of tapes at the crossing appear to be a suitable solution with a tensile stiffness reduction of $10 \%$ compared to the situation without cuts. These and other details and elements are tested to validate the final design. 
The use of reusable silicone vacuum bags was investigated in order to replace complex metal tooling blocks. Besides saving on mould materials and machining, the use of a preshaped silicone vacuum bag is expected to reduce labour cost due to a decrease in handling of tooling blocks for positioning and cleaning.

The first trial indicates that good quality laminates can be obtained using reusable vacuum bags. More testing will be carried out to validate the concept for a large $3 \times 1.2 \mathrm{~m}$ panel.

\section{ACKNOWLEDGEMENTS}

:* This project has received funding from the European Union's Horizon 2020 research and innovation programme under grant agreement No 723167.

\section{REFERENCES}

[1] H. Schinpers et al., "Broadband Conformal Phased Array with Optical Beam Forming for Airborne Satellite Communication”, IEEE Aerospace Conf., March 2008, Big Sky, MT, USA.

[2] S. Panthi, J. King, and C. McLain. "The eXConnect Broadband Aeronautical Service", 31st AIAA International Communications Satellite Systems Conference (ICSSC), (AIAA 20135621), October 14-17, 2013, Florence, Italy. https://doi.org/10.2514/6.2013-5621

[3] ACASIAS web site at http://www.acasias-project.eu/objectives-and-innovations-11, Accessed 21 March 2019.

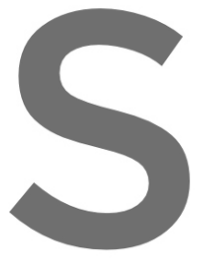

[4] Steven M. Huybre methods", ICCM12

[5] J. M. Müiller and stiffened composite panels manufactured usin Technical Conference, Long Beach, USA,
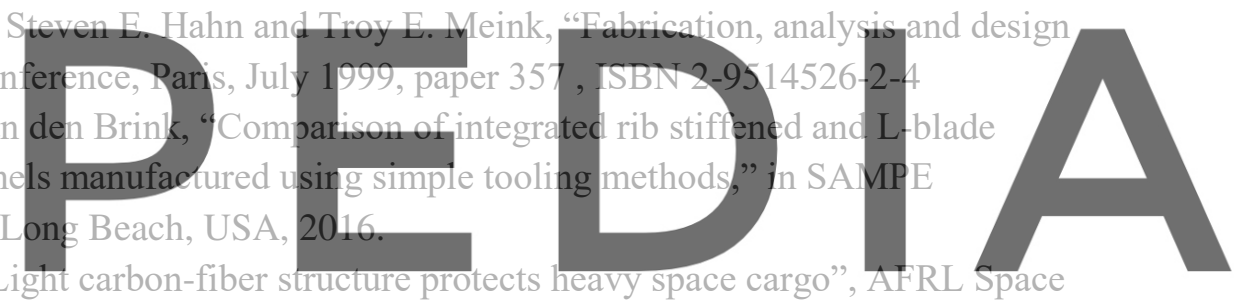

[6] Michael P. Kleiman, "Light carbon-fiber structure protec
Vehicles Directorate, February 26, 2007. Available at: 